\title{
Continuous solutions to Monge-Ampère equations on Hermitian manifolds for measures dominated by capacity
}

\author{
Sławomir Kołodziej ${ }^{1} \cdot$ Ngoc Cuong Nguyen $^{2}$
}

Received: 19 March 2020 / Accepted: 31 December 2020 / Published online: 27 April 2021

(c) The Author(s) 2021

\begin{abstract}
We prove the existence of a continuous quasi-plurisubharmonic solution to the MongeAmpère equation on a compact Hermitian manifold for a very general measure on the right hand side. We admit measures dominated by capacity in a certain manner, in particular, moderate measures studied by Dinh-Nguyen-Sibony. As a consequence, we give a characterization of measures admitting Hölder continuous quasi-plurisubharmonic potential, inspired by the work of Dinh-Nguyen.
\end{abstract}

Mathematics Subject Classification $32 \mathrm{U} 40 \cdot 32 \mathrm{~W} 20 \cdot 53 \mathrm{C} 55$

\section{Introduction}

Let $(X, \omega)$ be a compact Hermitian manifold of dimension $n$. The study of the complex Monge-Ampère equation in this setting was initiated by Cherrier [5], and the counterpart of the Calabi-Yau theorem [44] on compact Hermitian manifolds was proven by Tosatti and Weinkove [36]. Later Dinew and the authors, in a series of papers [11,24,26,27], obtained weak continuous solutions for more general densities on the right hand side of the equation, by extending the pluripotential methods employed before on Kähler manifolds. In this paper we deal with yet more general measures on the right hand side.

If $\omega$ is Kähler, then the first named author obtained in [20,21] the unique continuous $\omega$ plurisubharmonic ( $\omega$-psh for short) solution to the complex Monge-Ampère equation with the right hand side being a measure in one of the classes $\mathcal{F}(X, h)$ satisfying a bound in terms

Communicated by O. Savin.

$\triangle$ Sławomir Kołodziej

slawomir.kolodziej@im.uj.edu.pl

Ngoc Cuong Nguyen

cuongnn@kaist.ac.kr

1 Faculty of Mathematics and Computer Science, Jagiellonian University, Łojasiewicza 6, 30-348 Kraków, Poland

2 Department of Mathematical Sciences, KAIST, 291 Daehak-ro, Yuseong-gu, Daejeon 34141, South Korea 
of the Bedford-Taylor capacity and a weight function $h$ (the precise definition is given in the next section). We prove here the generalization of this result to Hermitian manifolds.

Theorem 1.1 Let $\mu \in \mathcal{F}(X, h)$ be such that $\mu(X)>0$. Then, there exist a continuous $\omega-p s h$ function $u$ and a constant $c>0$ solving the equation

$$
\left(\omega+d d^{c} u\right)^{n}=c \mu .
$$

If we assume further that the right hand side is strictly positive and absolutely continuous with respect to the Lebesgue measure, then we prove a stability of solutions and their uniqueness extending the main theorem of [27], (Theorem 4.1). Our method is adaptable to the Monge-Ampère type equations [32]. As a consequence, we get the existence and uniqueness of continuous $\omega$-psh solutions of these equations (Corollary 3.3).

The families of measures which belong to $\mathcal{F}(X, h)$, for some $h$, include those having densities in $L^{p}, p>1$, or even broader Orlicz spaces, but also measures singular with respect to $\omega^{n}$, for instance smooth forms on totally real submanifolds (see e.g. [2,20,42]). We shall distinguish classes $\mathcal{H}(\tau)$ which are unions (over $C>0$ ) of $\mathcal{F}\left(X, h_{1}\right)$ with $h_{1}(x)=C x^{n \tau}$ and fixed $\tau>0$; and $\mathcal{F}\left(X, h_{2}\right)$ with $h_{2}(x)=C e^{\alpha x}$ for some $C, \alpha>0$. The latter was introduced by Dinh, Nguyen and Sibony [12], who called the measures in this class (the union over $C>0, \alpha>0$ ) moderate. They proved that any measure locally dominated by the Monge-Ampère measure of a Hölder continuous psh function is moderate.

Later, Dinh and Nguyen [13] characterized the measures locally dominated by the MongeAmpère measure of a Hölder continuous psh function via the associated functionals acting on $\operatorname{PSH}(\omega)$ (the set of all $\omega$-psh functions) when $\omega$ is Kähler. In the last section we give a similar description in the Hermitian setting. Let us define

$$
\mathcal{S}:=\left\{v \in P S H(\omega):-1 \leq v \leq 0, \sup _{X} v=0\right\} .
$$

Let $\mu$ be a positive Radon measure on $X$ and $\hat{\mu}: P S H(\omega) \rightarrow \mathbb{R}$ the associated functional given by

$$
\hat{\mu}(v)=\int_{X} v d \mu
$$

Theorem 1.2 The measure $\mu$ belongs to $\mathcal{H}(\tau)$ and $\hat{\mu}$ is Hölder continuous with respect to $L^{1}$-distance on $\mathcal{S}$ if and only if there exist a Hölder continuous $\omega$-psh function $u$ and $a$ constant $c>0$ solving $\left(\omega+d d^{c} u\right)^{n}=c \mu$.

Notice that the Hölder continuity of $\hat{\mu}$ on the larger subset $\left\{v \in P S H(\omega): \sup _{X} v=0\right\}$ implies the $\mathcal{H}(\tau)$ property and the Hölder continuity on $\mathcal{S}$ (Proposition 5.5). The latter properties are independent. The examples [7, Example 5.5] or [13, Example 2.5] belong to $\mathcal{H}(\tau)$ for every $\tau>0$, but they do not admit Hölder continuous potentials. On the other hand, the well-known conjecture of Dinh, Nguyen, Sibony [13, Problem 1.5] predicted that the moderate property implies the Hölder continuity of the Monge-Ampère potential or equivalently the Hölder continuity on $\mathcal{S}$ of the functional associated to this measure.

As it was shown in [26] (inspired by [7]) that the existence of Hölder continuous solution is a local problem. We apply Theorem 1.2 to get main results of [35,42] in the Hermitian setting. For example, this gives a Hölder continuous $\omega$-psh potential for a smooth volume form of a compact smooth real hypersuface in $X$.

Let us indicate some motivations behind the study of the Monge-Ampère on Hermitian manifolds with measures on the right hand side. Unlike in the Kähler case, one solves the 
equation not only for a function but also for a constant on the right hand side. The range of those constants for a given manifold seems to have a geometrical meaning. It comes up in constructions of $\omega$ - psh functions with logarithmic poles like in [32,37] (where one solves the equation for approximants of Dirac measures); in connection to problems involving holomorphic Morse inequalities (see [29]), and others. Parabolic Monge-Ampère equations on a Hermitian manifold, the Chern-Ricci flow, are recently intensively studied (see [14$16,31,38-40,45])$. The flow is expected to play an important role in the classification of complex surfaces. In the context of parabolic equations the pluripotential estimates are also useful. For example, To [41] (independently, Nie [31] in particular cases) used results in [11,32] to prove a conjecture by Tosatti and Weinkove [38]. The geometric applications of pluripotential theory on Hermitian manifolds are discussed at length in surveys by Dinew $[9,10]$.

Another new topic is the complex dynamics on compact Hermitian manifolds. There the measures having interesting properties are often singular with respect to the volume form. In a recent paper $\mathrm{Vu}$ [43] showed that for any holomorphic dominant endomorphism $f$ of $X$ there exists an equilibrium measure $\mu_{f}$ associated to $f$. The understanding of this measure is a central problem in complex dynamics (as in the Kähler setting). By [43, Theorem 1.1] and Theorem 1.2 one gets that $\mu_{f}$ admits a Hölder continuous $\omega$-psh potential. We refer to [8] for results on the push-forwards of measures by dominant meromorphic maps between complex manifolds.

\section{Preliminaries}

In this section we recall and extend some results from [24,25,27]. Their statements are often more technical than the counterparts in the Kähler setting [22].

Let $h: \mathbb{R}_{+} \rightarrow(0, \infty)$ be an increasing function such that

$$
\int_{1}^{\infty} \frac{1}{x[h(x)]^{\frac{1}{n}}} d x<+\infty .
$$

In particular, $\lim _{x \rightarrow \infty} h(x)=+\infty$. Such a function $h$ is called admissible. In what follows we often omit to stress that $h$ is admissible. If $h$ is admissible, then so is $A_{2} h\left(A_{1} x\right)$ for every $A_{1}, A_{2}>0$. Define

$$
F_{h}(x)=\frac{x}{h\left(x^{-\frac{1}{n}}\right)} .
$$

Recall that the analogue of Bedford-Taylor capacity on compact complex manifolds is

$$
\operatorname{cap}_{\omega}(E):=\sup \left\{\int_{E} \omega_{w}^{n}: w \in \operatorname{PSH}(\omega), 0 \leq w \leq 1\right\},
$$

where $\operatorname{PSH}(\omega)$ is the set of $\omega$-psh functions on $X$ and $\omega_{w}^{n}:=\left(\omega+d d^{c} w\right)^{n}$. This capacity is equivalent to the Bedford-Taylor capacity [1] defined locally (see [22, page 52-53]).

Let $\mu$ be a positive Radon measure satisfying

$$
\mu(E) \leq F_{h}\left(\operatorname{cap}_{\omega}(E)\right),
$$

for any Borel set $E \subset X$ and some $F_{h}$.

Let us denote by $\mathcal{F}(X, h)$ the set of all measures that are dominated by the capacity cap $_{\omega}$ in the sense of (2.3) for some admissible $h$. 
Some particular families of measures which satisfy (2.3) were mentioned in Introduction. Another fairly general family is given in the following example. Note that these measures are often singular with respect to the Lebesgue measure and their potentials may not be Hölder continuous.

Example 2.1 ([28]) Let $\mu$ be a positive Borel measure such that it is locally dominated by Monge-Ampère measures of continuous plurisubharmonic functions whose modulus of continuity $\varpi(t)$ satisfy the Dini-type condition

$$
\int_{0}^{1} \frac{[\varpi(t)]^{\frac{1}{n}}}{t|\log t|} d t<+\infty .
$$

Then, $\mu \in \mathcal{F}(X, h)$ for some admissible function $h$.

Let us fix a finite covering of $X$ :

$$
\left\{B_{j}(s)\right\}_{j \in J} \text { where } B_{j}(s):=B\left(x_{j}, s\right)
$$

is the coordinate ball centered at $x_{j}$ of radius $s>0$. Take $s$ so small that $B\left(x_{j}, 3 s\right), j \in J$, are still coordinate balls. Let $\chi_{j}$ be the partition of unity subordinate to $\left.\left\{B_{j}(s)\right\}_{j \in J}\right\}$. The first observation is that if $\mu$ satisfies (2.3) on $X$, then in each chart $B_{j}(3 s)$ the same property holds for subsets of the smaller ball.

Lemma 2.2 Let $\mu \in \mathcal{F}(X, h)$. Then, for every compact $K \subset B_{j}(s) \subset \Omega:=B_{j}(3 s), j \in J$,

$$
\mu(K) \leq F_{h_{0}}(\operatorname{cap}(K, \Omega)) .
$$

for an admissible function $h_{0}$ depending only on $h, \omega, X$ and $\Omega$, where cap $(K, \Omega)$ is the relative capacity of Bedford and Taylor [1].

Proof The proof follows by the monotonicity of $h$ and the fact that

$$
\operatorname{cap}_{\omega}(K) \leq C_{1} \operatorname{cap}(K, \Omega),
$$

where $C_{1}$ is a uniform bound for plurisubharmonic functions on $B\left(x_{j}, 3 s\right)$ such that $v_{j}=0$ on $\partial \Omega$ and $d d^{c} v_{j} \geq \omega$ in $\Omega$ (see [22, page 53]). Thus, we can take

$$
h_{0}(x)=\frac{1}{C_{1}} h\left(C_{1}^{-\frac{1}{n}} x\right) \text {. }
$$

The proof is completed.

The second observation is the following.

Lemma 2.3 Let $\mu \in \mathcal{F}(X, h)$. Let $\mu_{U_{j}}$ be the restriction of $\chi_{j} \mu$ to the local coordinate $\Omega_{j}=B\left(x_{j}, 3 s\right) \subset \mathbb{C}^{n}$, where $U_{j}=B\left(x_{j}, s\right)$. Let $\rho_{\varepsilon}$ be the standard smoothing kernel on $B(0,3 s)$. Then,

$$
\mu_{\varepsilon}(z):=\sum_{j \in J} \mu_{U_{j}} * \rho_{\varepsilon}\left(z-x_{j}\right)
$$

is the sequence of smooth measures which converge weakly to $\mu$ as $\varepsilon$ tends to 0 . Moreover, $\mu_{\varepsilon} \in \mathcal{F}\left(X, h_{0}\right)$ for an admissible function $h_{0}$ when $\varepsilon$ is small enough. 
Proof Since the cover is finite, it is enough to show that each smooth measure of the right hand side belongs to $\mathcal{F}\left(\Omega, h_{0}\right)$ for an admissible function $h_{0}$. By Lemma 2.2 it follows that $\mu_{U_{j}} \in \mathcal{F}\left(\Omega, h_{0}\right)$. Thanks to [20, Eq.(3.5.1)] the convolutions with smoothing kernels preserve the inequality (2.6) when $\varepsilon$ is small enough.

We recall the basic result in [24]. Let $B>0$ be a constant such that

$$
-B \omega^{2} \leq 2 n d d^{c} \omega \leq B \omega^{2}, \quad-B \omega^{3} \leq 4 n^{2} d \omega \wedge d^{c} \omega \leq B \omega^{3} .
$$

Theorem 2.4 Fix $0<\varepsilon<1$. Let $\varphi, \psi \in \operatorname{PSH}(\omega) \cap L^{\infty}(X)$ be such that $\varphi \leq 0$, and $-1 \leq$ $\psi \leq 0 . \operatorname{Set} m(\varepsilon)=\inf _{X}[\varphi-(1-\varepsilon) \psi]$, and $\varepsilon_{0}:=\frac{1}{3} \min \left\{\varepsilon^{n}, \frac{\varepsilon^{3}}{16 B}, 4(1-\varepsilon) \varepsilon^{n}, 4(1-\varepsilon) \frac{\varepsilon^{3}}{16 B}\right\}$. Suppose that $\omega_{\varphi}^{n} \in \mathcal{F}(X, h)$. Then, for $0<t<\varepsilon_{0}$,

$$
t \leq \kappa\left[\operatorname{cap}_{\omega}(U(\varepsilon, t))\right],
$$

where $U(\varepsilon, t)=\{\varphi<(1-\varepsilon) \psi+m(\varepsilon)+t\}$, and the function $\kappa$ is defined on the interval $\left(0, \operatorname{cap}_{\omega}(X)\right)$ by the formula

$$
\kappa\left(s^{-n}\right)=4 C_{n}\left\{\frac{1}{[h(s)]^{\frac{1}{n}}}+\int_{s}^{\infty} \frac{d x}{x[h(x)]^{\frac{1}{n}}}\right\},
$$

with a dimensional constant $C_{n}$.

We use it to to generalize the stability estimate [26, Proposition 2.4] and [24, Corollary 5.10]. Let $\hbar(s)$ be the inverse function of $\kappa(s)$ and

$$
\Gamma(s) \text { the inverse function of } s^{n(n+2)+1} \hbar\left(s^{n+2}\right) .
$$

Notice that $\Gamma(s) \rightarrow 0$ as $s \rightarrow 0^{+}$.

Proposition 2.5 Let $\psi \in P S H(\omega) \cap C^{0}(X)$ and $\psi \leq 0$. Let $\mu \in \mathcal{F}(X, h)$. Assume that $\varphi \in P S H(\omega) \cap C^{0}(X)$ satisfies $\left(\omega+d d^{c} \varphi\right)^{n}=\mu$. Then, there exists a constant $C>0$ depending only on $\tau, \omega$ and $\|\psi\|_{L^{\infty}(X)}$ such that

$$
\sup _{X}(\psi-\varphi) \leq C \Gamma\left(\left\|(\psi-\varphi)_{+}\right\|_{L^{1}(d \mu)}\right) .
$$

Proof Without loss of generality we may assume that $-1 \leq \psi \leq 0$. Put

$$
U(\varepsilon, s)=\left\{\varphi<(1-\varepsilon) \psi+\inf _{X}[\varphi-(1-\varepsilon) \psi]+s\right\},
$$

where $0<\varepsilon<1$ and $s>0$.

Lemma 2.6 For $0<s \leq \frac{1}{3} \min \left\{\varepsilon^{n}, \frac{\varepsilon^{3}}{16 B}\right\}, 0<t \leq \frac{4}{3}(1-\varepsilon) \min \left\{\varepsilon^{n}, \frac{\varepsilon^{3}}{16 B}\right\}$ we have

$$
t^{n} \operatorname{cap}_{\omega(U(\varepsilon, s))} \leq C F_{h}\left(\operatorname{cap}_{\omega}(U(\varepsilon, s+t))\right),
$$

where $C$ is a dimensional constant.

Proof of Lemma 2.6 By [24, Lemma 5.4]

$$
t^{n} \operatorname{cap}_{\omega(U(\varepsilon, s))} \leq C \int_{U(\varepsilon, s+t)} \omega_{\varphi}^{n},
$$

The lemma follows from the assumption on the measure $\omega_{\varphi}^{n}=\mu$. 
To finish the proof of the proposition we proceed as in [26, Proposition 2.4] or [25, Theorem 3.11], though under a weaker assumption. One needs to estimate

$$
-S:=\sup _{X}(\psi-\varphi)>0
$$

in terms of $\left\|(\psi-\varphi)_{+}\right\|_{L^{1}(d \mu)}$ as in the Kähler case [21]. Suppose that

$$
\left\|(\psi-\varphi)_{+}\right\|_{L^{1}(d \mu)} \leq \delta,
$$

where $\delta:=\varepsilon^{n(n+2)+1} \hbar\left(\varepsilon^{n+2}\right)$. Consider sublevel sets $U(\varepsilon, t)=\left\{\varphi<(1-\varepsilon) \psi+S_{\varepsilon}+t\right\}$, where $S_{\varepsilon}=\inf _{X}[\varphi-(1-\varepsilon) \psi]$. It is clear that

$$
S-\varepsilon \leq S_{\varepsilon} \leq S
$$

Therefore, $U(\varepsilon, 2 t) \subset\{\varphi<\psi+S+\varepsilon+2 t\}$. Then, $(\psi-\varphi)_{+} \geq|S|-\varepsilon-2 t>0$ for $0<t<\varepsilon_{B}$ and $0<\varepsilon<|S| / 2$ on the latter set (if $|S| \leq 2 \varepsilon$ then we are done). By (2.11) we have

$$
\begin{aligned}
\operatorname{cap}_{\omega}(U(\varepsilon, t)) \leq \frac{C}{t^{n}} \int_{U(\varepsilon, 2 t)} d \mu & \leq \frac{C}{t^{n}} \int_{X} \frac{(\psi-\varphi)_{+}}{(|S|-\varepsilon-2 t)} d \mu \\
& =\frac{C\left\|(\psi-\varphi)_{+}\right\|_{L^{1}(d \mu)}}{t^{n}(|S|-\varepsilon-2 t)} .
\end{aligned}
$$

Moreover, by the inequality (2.8) it follows that $\hbar(t) \leq \operatorname{cap}_{\omega}(U(\varepsilon, t))$. Combining these inequalities, we obtain

$$
(|S|-\varepsilon-2 t) \leq \frac{C\left\|(\psi-\varphi)_{+}\right\|_{L^{1}(d \mu)}}{t^{n} \hbar(t)} .
$$

Therefore, using (2.12),

$$
\begin{aligned}
|S| & \leq \varepsilon+2 t+\frac{C\left\|(\psi-\varphi)_{+}\right\|_{L^{1}(d \mu)}}{t^{n} \hbar(t)} \\
& \leq 3 \varepsilon+\frac{C \delta}{t^{n} \hbar(t)} .
\end{aligned}
$$

Recall that $\varepsilon_{B}=\frac{1}{3} \min \left\{\varepsilon^{n}, \frac{\varepsilon^{3}}{16 B}\right\}$. So, taking $t=\varepsilon_{B} / 2 \geq \varepsilon^{n+2}$ we have

$$
\frac{\delta}{\varepsilon^{n(n+2)} \hbar\left(\varepsilon^{n+2}\right)}=\varepsilon .
$$

Notice that we used the fact that $\hbar(s)$ is also increasing. Hence $|S| \leq C \varepsilon$ with $C=C(\omega)$. Thus,

$$
\sup _{X}(\psi-\varphi) \leq C \Gamma\left(\left\|(\psi-\varphi)_{+}\right\|_{L^{1}(d \mu)}\right) .
$$

This is the desired stability estimate.

There is always a uniform lower bound for the volume of Monge-Ampère measures dominated by capacity. This is essentially [27, Proposition 2.4].

Proposition 2.7 Consider $\mu \in \mathcal{F}(X, h)$ such that $\mu(X)>0$. Suppose $w \in \operatorname{PSH}(\omega) \cap C(X)$ and $c>0$ solve

$$
\left(\omega+d d^{c} w\right)^{n}=c \mu, \quad \sup _{X} w=0,
$$


Then there exists a constant $V_{\min }>0$ depending only on $X, \omega, h$ such that whenever

$$
\int_{X} d \mu \leq 2 V_{\min }
$$

we have $c \geq 2^{n}$.

Proof Suppose $c \leq 2^{n}$. We shall see that this leads to a contradiction for some positive $V_{\min }$. Firstly, we have $\omega_{w}^{n} \leq 2^{n} \mu$. Therefore, the Monge-Ampère measure $\omega_{w}^{n}$ satisfies the inequality (2.3) for the admissible function $h(x) / 2^{n}$. The inequality (2.11) for $0<t \leq$ $\frac{1}{3} \min \left\{\frac{1}{2^{n}}, \frac{1}{2^{7} B}\right\}$ then gives:

$$
t^{n} \operatorname{cap}_{\omega}(\{w<S+t\}) \leq C \int_{\{w<S+2 t\}} \omega_{w}^{n} \leq C \int_{X} 2^{n} d \mu,
$$

where $S=\inf _{X} w$ and $C>0$ depends only on $n, B$. It implies that

$$
\frac{t^{n}}{2^{n} C} \operatorname{cap}_{\omega}(\{w<S+t\}) \leq \int_{X} d \mu .
$$

The formula (2.9) for the function $\kappa_{0}(x)$ corresponding to $\omega_{w}^{n}$ is

$$
\kappa_{0}\left(s^{-n}\right)=8 C_{n}\left\{\frac{1}{[h(s)]^{\frac{1}{n}}}+\int_{s}^{\infty} \frac{d x}{x[h(x)]^{\frac{1}{n}}}\right\} .
$$

It is defined on $\left(0, \operatorname{cap}_{\omega}(X)\right)$. Since $\kappa_{0}(x)$ is an increasing function it has the inverse $\hbar_{0}(x)$. It follows from (2.8) that for $0<t \leq \frac{1}{3} \min \left\{\frac{1}{2^{n}}, \frac{1}{2^{7} B}\right\}$ we have

$$
\hbar_{0}(t) \leq \operatorname{cap}_{\omega}(\{w<S+t\}) .
$$

Coupling this with (2.15) we obtain

$$
\int_{X} d \mu \geq \frac{t^{n} \hbar_{0}(t)}{2^{n} C}
$$

Define

$$
V_{\text {min }}:=\frac{t_{0}^{n}}{2^{n+2} C \hbar_{0}\left(t_{0}\right)}>0, \quad t_{0}=\frac{1}{6} \min \left\{\frac{1}{2^{n}}, \frac{1}{2^{7} B}\right\} .
$$

Then, (2.16) and the above choices lead to a contradiction

$$
2 V_{\text {min }} \geq \int_{X} d \mu \geq 4 V_{\text {min }}>0 .
$$

Thus the proposition is proven.

\section{Existence of continuous solutions}

In this section we generalize the results of $[24,26]$ on the existence of continuous solutions of the Monge-Ampère equation. This is also the extension of [20,22] from Kähler to Hermitian setting. We prove the first theorem in the introduction.

Theorem 3.1 Let $\mu \in \mathcal{F}(X, h)$ be such that $\mu(X)>0$. Then, there exists a continuous $\omega$-psh function $u$ and a constant $c>0$ solving the equation

$$
\left(\omega+d d^{c} u\right)^{n}=c \mu .
$$


Proof The proof follows the scheme of the one in [26, Theorem 1.3]. We only clarify the differences. Let $\mu_{\varepsilon}$ be the approximating sequence from Lemma 2.3. By [24, Theorem 0.1] there exist $u_{\varepsilon} \in P S H(\omega) \cap C^{0}(X)$ and a constant $c_{\varepsilon}>0$ solving

$$
\left(\omega+d d^{c} u_{\varepsilon}\right)^{n}=c_{\varepsilon} \mu_{\varepsilon}, \quad \sup _{X} u_{\varepsilon}=0 .
$$

The main difficulty lies in proving the uniform upper bound for constants $\left\{c_{\epsilon}\right\}$ which requires a bit different approach compared to [24,26].

Since $\lim _{\varepsilon \rightarrow 0} \mu_{\varepsilon}(X)=\mu(X)$, there exist a ball $U=B(a, s) \subset U^{\prime}=B(a, 2 s)$ in the finite open cover (2.5) and a positive constant $C_{1}>0$ such that

$$
\mu_{U} * \varrho_{\varepsilon}(X)=\mu_{U} * \varrho_{\varepsilon}\left(U^{\prime}\right) \geq C_{1}
$$

for every small $\varepsilon>0$, where we recall $\mu_{U}$ is the restriction of $\chi_{a} \mu$ to $U$, and $\chi_{a}$ is the smooth function in the partition of unity subordinate to $\left\{B\left(x_{j}, s\right)\right\}$. Let us denote $\Omega=B(a, 3 s)$. Thanks to [3] there is $v_{\varepsilon} \in P S H(\Omega) \cap C^{\infty}(\bar{\Omega})$ such that $\left(d d^{c} v_{\varepsilon}\right)^{n}=\mu_{U} * \varrho_{\varepsilon}+\varepsilon \omega^{n}$ and $v_{\varepsilon}=0$ on $\partial \Omega$. By Lemma 2.3 and [19] it follows that

$$
\left\|v_{\varepsilon}\right\|_{L^{\infty}(\Omega)} \leq C_{2}=C\left(\Omega, h_{0}\right) .
$$

It is clear that $\mu_{\varepsilon} \geq \mu_{U} * \varrho_{\varepsilon}$ on $\Omega$. Let us write $\mu_{U} * \varrho_{\varepsilon}=R_{\varepsilon} \omega^{n}$ for a smooth function $R_{\varepsilon}$ in $\Omega$. Using the mixed forms type inequality [27, Lemma 2.2] we have

$$
\begin{aligned}
\omega_{u_{\varepsilon}} \wedge\left(d d^{c} v_{\varepsilon}\right)^{n-1} & \geq\left(\frac{\omega_{u_{\varepsilon}}^{n}}{\left(d d^{c} v_{\varepsilon}\right)^{n}}\right)^{\frac{1}{n}}\left(d d^{c} v_{\varepsilon}\right)^{n} \\
& \geq\left(\frac{c_{\varepsilon} R_{\varepsilon}}{R_{\varepsilon}+\varepsilon}\right)^{\frac{1}{n}}\left(R_{\varepsilon}+\varepsilon\right) \omega^{n} \\
& \geq c_{\varepsilon}^{\frac{1}{n}} R_{\varepsilon} \omega^{n} .
\end{aligned}
$$

Therefore,

$$
\int_{\Omega^{\prime}} \omega_{u_{\varepsilon}} \wedge\left(d d^{c} v_{\varepsilon}\right)^{n-1} \geq c_{\varepsilon}^{\frac{1}{n}} C_{1} .
$$

Fix a strictly plurisubharmonic function $\rho_{\Omega}$ in $\Omega$ such that $\omega \leq d d^{c} \rho_{\Omega}$. Then the Demailly's version of the Chern-Levine-Nirenberg inequality [6] gives

$$
\begin{aligned}
\int_{\Omega^{\prime}} \omega_{u_{\varepsilon}} \wedge\left(d d^{c} v_{\varepsilon}\right)^{n-1} & \leq \int_{\Omega^{\prime}} d d^{c}\left(u+\rho_{\Omega}\right) \wedge\left(d d^{c} v_{\varepsilon}\right)^{n-1} \\
& \leq C\left(\Omega^{\prime}, \Omega\right)\left\|v_{\varepsilon}\right\|_{L^{\infty}(\Omega)}^{n-1}\left(\left\|\rho_{\Omega}\right\|_{L^{1}(\Omega)}+\left\|u_{\varepsilon}\right\|_{L^{1}(\Omega)}\right)
\end{aligned}
$$

Notice that $\int_{X}\left|u_{\varepsilon}\right| \omega^{n}$ is uniformly bounded (see e.g. [11, Proposition 2.5]). These combined with (3.2) and (3.3) give the uniform upper bound for $\left\{c_{\varepsilon}\right\}_{\varepsilon>0}$. The uniform lower bound of this sequence follows from [24, Lemma 5.9] as $\mu_{\varepsilon}(X)$ is uniformly bounded. By the proof of [24, Corollary 5.6] it follows

$$
\left\|u_{\varepsilon}\right\|_{L^{\infty}(X)}<C .
$$

Now we continue as in the proof of [26, Theorem 1.3]. Since the sequence $\left\{u_{\varepsilon}\right\}_{\varepsilon>0}$ normalized by $\sup _{X} u_{\varepsilon}=0$ is a compact subset of $L^{1}(X)$, passing to a subsequence, we may assume that

$$
u_{\varepsilon} \longrightarrow u \text { in } L^{1}(X) \text {; }
$$


moreover, $u \in P S H(\omega) \cap L^{\infty}(X)$ and also $\lim _{\varepsilon \rightarrow 0} c_{\varepsilon}=c>0$.

We wish to apply Proposition 2.5 to conclude that the convergence (3.4) is in $C^{0}(X)$. This amounts to showing that

$$
\lim _{\varepsilon \rightarrow 0} \int_{X}\left|u_{\varepsilon}-u\right| d \mu_{\varepsilon}=0 .
$$

By (2.3) the measure $\mu$ satisfies

$$
\mu(K) \leq \operatorname{Acap}_{\omega}(K)
$$

for all Borel sets $K \subset X$, where $A$ is a uniform constant. Furthermore, the potentials are uniformly bounded, so we can repeat the arguments of [18, Lemma 4.4] (see also [4, Lemma 5.2 and Proof of Theorem 5.1]) to finish the proof of (3.5). Finally, we get that $u_{\varepsilon}$ converges to $u$ in $C^{0}(X)$, which is a solution to $\omega_{u}^{n}=c \mu$.

Corollary 3.2 Suppose that $\mu_{j} \in \mathcal{F}(X, h)$ and it is smooth for every $j \geq 1$. Let $\mu_{j}$ converge weakly to $\mu \in \mathcal{F}(X, h)$ as $j \rightarrow+\infty$. For $j \geq 1$ let us solve

$$
\left(\omega+d d^{c} v_{j}\right)^{n}=e^{v_{j}} \mu_{j} .
$$

Then $v_{j}$ converges uniformly to a continuous $\omega$-psh function $v$ as $j \rightarrow+\infty$. Consequently, $v$ is the unique continuous $\omega$-psh solution to $\omega_{v}^{n}=e^{v} \mu$.

Proof With the estimates (3.1)-(3.3) at hand the proof of [32, Theorem 2.1] is readily adaptable to this setting which gives the existence of a continuous solution. The uniqueness follows from [32, Lemma 2.3] with the same proof.

Corollary 3.3 Let $\mu \in \mathcal{F}(X, h)$ and $\lambda>0$. Then, there exists a unique continuous $\omega-p s h$ solution $v$ to

$$
\left(\omega+d d^{c} v\right)^{n}=e^{\lambda v} \mu
$$

Proof It is a simple application of Corollary 3.2 for the approximating sequence $\mu_{\varepsilon}$ from Lemma 2.3.

\section{Stability of solutions}

We prove a stability estimate for measures belonging to $\mathcal{F}(X, h)$ which are strictly positive, absolutely continuous with respect to the Lebesgue measure. We use the following notation: the $L^{p}$-norms for $0<p<\infty$ are

$$
\|\cdot\|_{p}:=\left(\int_{X}|\cdot|^{p} \omega^{n}\right)^{\frac{1}{p}} \text { and }\|\cdot\|_{\infty}:=\sup _{X}|\cdot| .
$$

Theorem 4.1 Assume $f, g \in L^{1}(X)$ and $f \omega^{n}, g \omega^{n} \in \mathcal{F}(X, h)$. Consider two bounded $\omega$ psh solutions $u, v$ of

$$
\omega_{u}^{n}=f \omega^{n}, \quad \omega_{v}^{n}=g \omega^{n}
$$

with $\sup _{X} u=\sup _{X} u=0$. Suppose that $f \geq c_{0}>0$. Fix $\gamma>2+n(n+1)$. Then,

$$
\|u-v\|_{\infty} \leq C \varepsilon
$$


provided that

$$
\|f-g\|_{1} \leq \hbar\left(\varepsilon^{n+1}\right) \varepsilon^{\gamma} .
$$

Remark 4.2 Very recently Lu et al. [30] improved the stability estimates for $f, g \in L^{p}(X)$ with $p>1$, with the same exponent as in the Kähler case, by a clever use of the stability estimate for Monge-Ampère type equation from the Guedj et al. work [17]. Given the existence of solution in Corollary 3.3 for measures belonging to $\mathcal{F}(X, h)$ with $L^{1}$-densities we expect that the stability estimate above can be improved.

We will adapt the proof of [27, Theorem 3.1] with necessary changes. First, it is enough to assume that $f, g$ are smooth.

Lemma 4.3 Let $f_{j}, g_{j} \in \mathcal{F}(X, h)$ be smooth sequences of functions converging in $L^{1}(X)$ to $f, g$ respectively. Let $u_{j}, v_{j} \in P S H(\omega) \cap C^{\infty}(X)$ be such that $u_{j} \searrow u$ and $v_{j} \searrow u$ as $j \rightarrow+\infty$. Assume $\varphi_{j}, \psi_{j}$ solve

$$
\left(\omega+d d^{c} \varphi_{j}\right)^{n}=e^{\varphi_{j}-u_{j}} f_{j} \omega^{n}, \quad\left(\omega+d d^{c} \psi_{j}\right)^{n}=e^{\psi_{j}-v_{j}} g_{j} \omega^{n} .
$$

Then,

$$
\|u-v\|_{\infty}=\lim _{j \rightarrow+\infty}\left\|\varphi_{j}-\psi_{j}\right\|_{\infty}
$$

Proof We use the argument of [27, Remark 3.11] pointed out by a referee of that paper. By Corollary 3.2 the sequence $\left\{\varphi_{j}\right\}$ converges uniformly to the solution $u_{0}$ of

$$
\left(\omega+d d^{c} u_{0}\right)^{n}=e^{u_{0}}\left(e^{-u} f\right) \omega^{n} .
$$

It follows from the uniqueness of $u$ that $u_{0}=u$. Similarly, $\left\{\psi_{j}\right\}$ converges uniformly to $v$. The conclusion follows.

Proof of Theorem 4.1 We fix the notation as in the proof of [27, Theorem 3.1]. For $t \in \mathbb{R}$ define

$$
\varphi=u-v, \quad \Omega(t)=\{\varphi<t\}, \quad t_{0}=\inf _{X} \varphi .
$$

We need to replace [27, Lemma 3.4] by the following statement. The proof is similar up to some technicalities. For the reader's convenience we give all details here.

Lemma 4.4 Let $V_{\min }>0$ be the constant in Proposition 2.7. Fix $t_{1}>t_{0}$. Assume that for $0<\varepsilon<<1$,

$$
\|f-g\|_{1} \leq \ell(\varepsilon) \varepsilon
$$

where $\ell(\varepsilon)=\hbar\left(\varepsilon^{(n+1) \alpha}\right)$. If $\int_{\Omega\left(t_{1}\right)} f \omega^{n} \leq V_{\min }$, then

$$
t_{1}-t_{0} \leq C \varepsilon^{\alpha}
$$

where $0<\alpha<\frac{1}{2+n(n+1)}$ is fixed.

Proof Define the sets:

$$
\Omega_{1}:=\left\{z \in \Omega\left(t_{1}\right): f(z) \leq\left(1+\varepsilon^{\alpha}\right) g(z)\right\} \text { and } \quad \Omega_{2}:=\Omega\left(t_{1}\right) \backslash \Omega_{1} .
$$


Since $g<\varepsilon^{-\alpha}(f-g)$ on $\Omega_{2}$, we have

$$
\begin{aligned}
\int_{\Omega_{2}} f \omega^{n} & \leq \int_{\Omega_{2}}|f-g| \omega^{n}+\int_{\Omega_{2}} g \omega^{n} \\
& \leq \ell(\varepsilon) \varepsilon+\ell(\varepsilon) \varepsilon^{1-\alpha} \\
& \leq 2 \ell(\varepsilon) \varepsilon^{1-\alpha} .
\end{aligned}
$$

It follows that

$$
\int_{\Omega\left(t_{1}\right)} f \omega^{n}=\int_{\Omega_{1}} f \omega^{n}+\int_{\Omega_{2}} f \omega^{n} \leq \int_{\Omega_{1}} f \omega^{n}+2 \ell(\varepsilon) \varepsilon^{1-\alpha} \leq V_{\min }+2 \ell(\varepsilon) \varepsilon^{1-\alpha} .
$$

Next, we construct a barrier function by putting

$$
\hat{f}(z)= \begin{cases}f(z) & \text { for } z \in \Omega\left(t_{1}\right) \\ \frac{1}{A} f(z) & \text { for } z \in X \backslash \Omega\left(t_{1}\right)\end{cases}
$$

As $\int_{\Omega\left(t_{1}\right)} f \omega^{n} \leq V_{\min }$ we can choose $A>1$ large enough so that

$$
\int_{X} \hat{f} \omega^{n} \leq \frac{3}{2} V_{\min }
$$

Notice that $f / A \leq \hat{f} \leq f$. By Theorem 3.1 we find $w \in P S H(\omega) \cap C(X)$ and $\hat{c}>0$ satisfying

$$
\left(\omega+d d^{c} w\right)^{n}=\hat{c} \hat{f} \omega^{n}, \quad \sup _{X} w=0 .
$$

By Proposition 2.7 applied for $f, h$ we have

$$
2^{n} \leq \hat{c} \leq A,
$$

where the last inequality follows from (4.2) and [27, Lemma 2.1]. Hence,

$$
\hat{c} \hat{f} \geq 2^{n} f \text { on } \Omega\left(t_{1}\right) .
$$

Define for $0<s<1, \psi_{s}=(1-s) v+s w$. It follows from the mixed forms type inequality ([27, Lemma 2.2]) that

$$
\begin{aligned}
\left(\omega+d d^{c} \psi_{s}\right)^{n} & \geq\left[(1-s) g^{\frac{1}{n}}+s(\hat{c} \hat{f} / f)^{\frac{1}{n}}\right]^{n} \omega^{n} \\
& =\left[(1-s)(g / f)^{\frac{1}{n}}+s(\hat{c} \hat{f} / f)^{\frac{1}{n}}\right]^{n} f \omega^{n} \\
& =:[b(s)]^{n} f \omega^{n} .
\end{aligned}
$$

Therefore on $\Omega_{1}$ we have

$$
b(s) \geq \frac{(1-s)}{\left(1+\varepsilon^{\alpha}\right)^{\frac{1}{n}}}+2 s \geq \frac{1-s}{1+\varepsilon^{\alpha}}+2 s .
$$

If $2 \varepsilon^{\alpha} \leq s \leq 1$, then

$$
b(s) \geq 1+\varepsilon^{\alpha} \text { on } \Omega_{1} .
$$

Let us use the notation $m_{s}:=\inf _{X}\left(u-\psi_{s}\right)=\inf _{X}\{u-v+s(v-w)\}$. Then,

$$
m_{s} \leq t_{0}+s\|w\|_{\infty} .
$$


Set for $0<\tau<1, m_{S}(\tau):=\inf _{X}\left[u-(1-\tau) \psi_{s}\right]$. Then $m_{S}(\tau) \leq m_{s}$. By the above definitions we have

$$
\begin{aligned}
U(\tau, t) & :=\left\{u<(1-\tau) \psi_{s}+m_{s}(\tau)+t\right\} \\
& \subset\left\{u<\psi_{s}+m_{s}+\tau\left\|\psi_{s}\right\|_{\infty}+t\right\} \\
& \subset\left\{u<v+t_{0}+s\left(\|v\|_{\infty}+\|w\|_{\infty}\right)+\tau\left\|\psi_{s}\right\|_{\infty}+t\right\} .
\end{aligned}
$$

We are going to show that

$$
t_{1}-t_{0} \leq 2 s\left(\|v\|_{\infty}+\|w\|_{\infty}\right)+\tau\left\|\psi_{s}\right\|_{\infty},
$$

for $s=2 \varepsilon^{\alpha}$ and $\tau=\varepsilon^{\alpha} / 2$. Suppose it was false. By (4.6) we have

$$
U(\tau, t) \subset \subset\left\{u<v+t_{0}+\left(t_{1}-t_{0}\right)\right\}=\Omega\left(t_{1}\right),
$$

for $0<t<\frac{t_{1}-t_{0}}{2}$. To go further we need to estimate the integrals:

$$
\int_{U(\tau, t)} f \omega^{n}
$$

for $0<t<<s, \tau$. By the modified comparison principle [24, Theorem 0.2]

$$
\int_{U(\tau, t)} \omega_{(1-\tau) \psi_{s}}^{n} \leq\left(1+\frac{C t}{\tau^{n}}\right) \int_{U(\tau, t)} \omega_{u}^{n},
$$

for every $0<t<\min \left\{\frac{\tau^{3}}{16 B}, \frac{t_{1}-t_{0}}{2}\right\}$. Hence, a simple estimate from below gives

$$
(1-\tau)^{n} \int_{U(\tau, t)} \omega_{\psi_{s}}^{n} \leq\left(1+\frac{C t}{\tau^{n}}\right) \int_{U(\tau, t)} \omega_{u}^{n} .
$$

Using (4.5) for $s=2 \varepsilon^{\alpha}$ we get

$$
(1-\tau)^{n}\left(1+\varepsilon^{\alpha}\right)^{n} \int_{U(\tau, t) \cap \Omega_{1}} f \omega^{n} \leq\left(1+\frac{C t}{\tau^{n}}\right) \int_{U(\tau, t)} f \omega^{n} .
$$

If we write $a(\varepsilon, \tau)=(1-\tau)^{n}\left(1+\varepsilon^{\alpha}\right)^{n}$, then

$$
a(\varepsilon, \tau)=\left(1+\varepsilon^{\alpha} / 2-\varepsilon^{2 \alpha} / 2\right)^{n}>1+\varepsilon^{\alpha} / 4
$$

as we have $\tau=\varepsilon^{\alpha} / 2$ and $0<\varepsilon^{\alpha}<1 / 4$. Therefore (4.8) implies that

$$
\left[a(\varepsilon, \tau)-\left(1+\frac{2^{n} C t}{\varepsilon^{n \alpha}}\right)\right] \int_{U(\tau, t) \cap \Omega_{1}} f \omega^{n} \leq\left(1+\frac{2^{n} C t}{\varepsilon^{n \alpha}}\right) \int_{\Omega_{2}} f \omega^{n} .
$$

Thus for $0<t \leq \varepsilon^{(n+1) \alpha} / 2^{n+3} C$,

$$
\frac{\varepsilon^{\alpha}}{8} \int_{U(\tau, t) \cap \Omega_{1}} f \omega^{n} \leq 2 \int_{\Omega_{2}} f \omega^{n} \leq 4 \ell(\varepsilon) \varepsilon^{1-\alpha},
$$

where the last inequality used (4.1). Hence,

$$
\int_{U(\tau, t) \cap \Omega_{1}} f \omega^{n} \leq 32 \ell(\varepsilon) \varepsilon^{1-2 \alpha} .
$$

Altogether we get that for $0<t \leq \varepsilon^{(n+1) \alpha} / C$,

$$
\int_{U(\tau, t)} f \omega^{n} \leq \int_{U(\tau, t) \cap \Omega_{1}} f \omega^{n}+\int_{\Omega_{2}} f \omega^{n} \leq C \ell(\varepsilon) \varepsilon^{1-2 \alpha} .
$$


This is the estimate we need.

Now we are able make use of the results from [24] recalled above. First, it follows from (2.8) and (2.11) that for $0<t \leq \varepsilon^{(n+1) \alpha} / C$,

$$
\hbar(t / 2) \leq \operatorname{cap}_{\omega}(U(\tau, t / 2)) \leq \frac{2^{n} C}{t^{n}} \int_{U(\tau, t)} f \omega^{n},
$$

where $\hbar(t)$ is the inverse of $\kappa(t)$. It follows from (4.9) and (4.10) that

$$
\hbar(t) \leq \frac{C \ell(\varepsilon) \varepsilon^{1-2 \alpha}}{t^{n}} .
$$

Then, taking $t=\varepsilon^{(n+1) \alpha}$ we obtain that

$$
\hbar\left(\varepsilon^{(n+1) \alpha}\right) \leq C \hbar\left(\varepsilon^{(n+1) \alpha}\right) \varepsilon^{1-2 \alpha-n(n+1) \alpha} .
$$

Equivalently, $1 \leq C \varepsilon^{1-2 \alpha-n(n+1) \alpha}$. However, we have that $1-[n(n+1)+2] \alpha>0$, which leads to a contradiction for $\varepsilon>0$ small enough.

Thus we have proved that

$$
t_{1}-t_{0} \leq 4 \varepsilon^{\alpha}\left(\|v\|_{\infty}+\|w\|_{\infty}+\left\|\psi_{s}\right\|_{\infty}\right),
$$

for a fixed $0<\alpha<\frac{1}{2+n(n+1)}$. The norms on the right hand side are controlled by $\|f\|_{1},\|g\|_{1}, h, V_{\min }$. So the lemma follows by rewriting $\gamma=1 / \alpha$ and $\varepsilon:=\varepsilon^{1 / \alpha}$.

Thanks to the above lemma, the remaining part of the proof of [27, Theorem 3.1] is used to conclude that of Theorem 4.1 .

\section{The Dinh-Nguyen theorem on Hermitian manifolds}

In this section we give a characterization of measures leading to Hölder continuous solutions of the Monge-Ampère equation on compact Hermitian manifolds, which is an analogue of the Dinh-Nguyen theorem [13]. If $\omega$ is Kähler, [13] says that a positive Radon measure admits a Hölder continuous $\omega$-psh potential if and only if the associated functional is Hölder continuous on $\left\{w \in \operatorname{PSH}(\omega): \sup _{X} v=0\right\}$ with respect to the $L^{1}$-distance. Let us denote

$$
\mathcal{S}=\mathcal{S}(\omega):=\left\{u \in P S H(\omega):-1 \leq u \leq 0, \sup _{X} u=0\right\} .
$$

The $L^{1}$-distance, with respect to the Lebesgue measure, between $u, v \in P S H(\omega)$ is given by

$$
\|u-v\|_{L^{1}}:=\int_{X}|u-v| \omega^{n} .
$$

A measure $\mu$ gives the natural functional $\hat{\mu}: P S H(\omega) \rightarrow \mathbb{R}$ defined by

$$
\hat{\mu}(v)=\int_{X} v d \mu .
$$

Following Dinh-Nguyen [13] we say that

Definition 5.1 $\hat{\mu}$ is Hölder continuous on $\mathcal{S}$ if it is Hölder continuous with respect to the $L^{1}$ distance. 
In other words there exist a uniform exponent $\alpha>0$ and a uniform constant $C>0$ such that for every $u, v \in \mathcal{S}$,

$$
|\hat{\mu}(u-v)|=\left|\int_{X}(u-v) d \mu\right| \leq C\|u-v\|_{L^{1}}^{\alpha} .
$$

Since $\max \{u, v\} \in \mathcal{S}$ for every $u, v \in \mathcal{S}$, this inequality is equivalent to

$$
\int_{X}|u-v| d \mu \leq C\|u-v\|_{L^{1}}^{\alpha} \quad \forall u, v \in \mathcal{S} .
$$

We are going to show that the Hölder continuity property on $\mathcal{S}$ is local. Let $\Omega$ be a strictly pseudoconvex domain in $\mathbb{C}^{n}$ and define

$$
\mathcal{S}_{0}(\Omega):=\{v \in \operatorname{PSH}(\Omega):-1 \leq v \leq 0\} .
$$

The $L^{1}$ distance (with respect to the Lebesgue measure) between $\varphi, \psi \in \mathcal{S}_{0}$ is defined similarly:

$$
\|\varphi-\psi\|_{L^{1}(\Omega)}=\int_{\Omega}|\varphi-\psi| d V_{2 n} .
$$

Let $v$ be a positive Borel measure on $\Omega$. It also gives a natural functional $\hat{v}$ on $\operatorname{PSH}(\Omega)$ defined by

$$
\hat{v}(\varphi)=\int_{\Omega} \varphi d \nu
$$

Definition $5.2 \hat{v}$ is locally Hölder continuous on $\mathcal{S}_{0}(\Omega)$ if for a fixed $\Omega^{\prime} \subset \subset \Omega$, there exists a constant $C=C\left(\Omega^{\prime}, \Omega\right)>0$ and an exponent $\alpha>0$ such that for every $\varphi, \psi \in \mathcal{S}_{0}(\Omega)$

$$
\int_{\Omega^{\prime}}|\varphi-\psi| d \nu \leq C\|\varphi-\psi\|_{L^{1}(\Omega)}^{\alpha} .
$$

Lemma 5.3 Let $\mu$ be a positive Borel measure on X. Then, $\hat{\mu}$ is Hölder continuous on $\mathcal{S}$ if and only if it is locally Hölder continuous on every local coordinate chart.

Proof Suppose that $\hat{\mu}$ is locally Hölder continuous on each local coordinate chart. Let $u, v \in$ $\mathcal{S}$. We wish to show that there exist $C, \alpha>0$ such that

$$
\int_{X}|u-v| d \mu \leq C\|u-v\|_{L^{1}}^{\alpha} .
$$

Let $B(a, r)$ be a local coordinate ball in the finite covering (2.5). Let $\rho$ be a strictly plurisubharmonic function on $U:=B(a, 2 r)$ such that $d d^{c} \rho \geq \omega$. Define $\varphi:=u+\rho, \psi:=v+\rho$. By local Hölder continuity of $\hat{\mu}$ we have

$$
\int_{B(a, r)}|u-v| d \mu=\int_{B(a, r)}|\varphi-\psi| d \mu \leq C\|\varphi-\psi\|_{L^{1}(U)}^{\alpha} \leq C\left(\int_{X}|u-v| \omega^{n}\right)^{\alpha} .
$$

Summing up over all $j \in J$ of the cover, we get that $\hat{\mu}$ is Hölder continuous on $\mathcal{S}$.

For the reverse direction, assume now that $\hat{\mu}$ is Hölder continuous $\mathcal{S}$. Let $B(a, r), U$ be the coordinate balls above. Take $\varphi, \psi \in \mathcal{S}_{0}(U)$. Let $\chi$ be a $\omega$-psh function on $X$ such that $\chi=0$ outside $U$ and $\chi \leq-3 \delta$ on $B(a, r)$ for some $0<\delta<1 / 2$. Define

$$
\tilde{\varphi}= \begin{cases}\max \{\delta \varphi-\delta, \chi\} & \text { on } U, \\ \chi & \text { on } X \backslash U,\end{cases}
$$


and $\tilde{\psi}$ analogously. Then, using the assumption

$$
\delta \int_{B(a, r)}|\varphi-\psi| d \mu \leq \int_{X}|\tilde{\varphi}-\tilde{\psi}| d \mu \leq C\left(\int_{X}|\tilde{\varphi}-\tilde{\psi}| \omega^{n}\right)^{\alpha}=C\left(\int_{U}|\tilde{\varphi}-\tilde{\psi}| \omega^{n}\right)^{\alpha} .
$$

Note that on $U$ we have $|\tilde{\varphi}-\tilde{\psi}| \leq \delta|\varphi-\psi|$. It follows that

$$
\int_{B(a, r)}|\varphi-\psi| d \mu \leq \frac{C}{\delta^{1-\alpha}}\left(\int_{U}|\varphi-\psi| \omega^{n}\right)^{\alpha} .
$$

This is the local Hölder continuous property of $\hat{\mu}$ on $U$.

There are plenty of examples of measures which are locally Hölder continuous on $\mathcal{S}_{0}(\Omega)$ (see $[33,34])$. We give below a sufficient condition. Let us consider the class

$$
\mathcal{E}_{0}^{\prime}(\Omega)=\left\{v \in P S H \cap L^{\infty}(\Omega): \lim _{z \rightarrow \partial \Omega} v(z)=0, \int_{\Omega}\left(d d^{c} v\right)^{n} \leq 1\right\} .
$$

Then, the Hölder continuity of a functional on $\mathcal{E}_{0}^{\prime}(\Omega)$ is considered with respect to $L^{1}$-distance [33, Definition 2.3].

Lemma 5.4 If $\hat{v}$ is Hölder continuous on $\mathcal{E}_{0}^{\prime}(\Omega)$, then it is locally Hölder continuous on $\mathcal{S}_{0}(\Omega)$.

Proof Let $\Omega^{\prime} \subset \subset \Omega$ and $u, v \in \mathcal{S}_{0}(\Omega)$. Let $\rho$ be the defining function of $\Omega$. By the maximum construction we may assume that there are $\tilde{u}, \tilde{v} \in \operatorname{PSH}(\Omega)$ such that

$$
\tilde{u}=u, \tilde{v}=v \text { in } \Omega^{\prime}
$$

and $\tilde{u}=\tilde{v}=\rho$ near $\partial \Omega$. The Chern-Levin-Nirenberg inequality implies that $\tilde{u} / c_{0}, \tilde{v} / c_{0} \in$ $\mathcal{E}_{0}^{\prime}(\Omega)$ for a constant $c_{0}>0$ depending only on $\rho$ and $\Omega^{\prime}, \Omega$. Thus,

$$
\int_{\Omega^{\prime}}|u-v| d v \leq \int_{\Omega}|\tilde{u}-\tilde{v}| d v \leq C c_{0}\|\tilde{u}-\tilde{v}\|_{L^{1}(\Omega)}^{\alpha} \leq C c_{0}\|u-v\|_{L^{1}(\Omega)}^{\alpha},
$$

where the last inequality used the fact that $|\tilde{u}-\tilde{v}| \leq|u-v|$ in $\Omega$.

Let us consider the following classes of measures:

$$
\mathcal{H}(\tau)=\left\{\mu \in \mathcal{F}\left(X, h_{1}\right): h_{1}(x)=C_{1} x^{n \tau} \text { for some } C_{1}, \tau>0\right\},
$$

and the moderate measures, which by definition, are in $\mathcal{F}\left(X, h_{2}\right)$ with $h_{2}(x)=C_{2} e^{\alpha x}$ for some $C_{2}, \alpha>0$. For the latter the stability estimate of its potential has a nicer form, i.e., the function defined in (2.10) is

$$
\Gamma(s)=C s^{\alpha} \quad \text { with } \alpha>0 .
$$

We observe that the proof of [13, Proposition 4.4] holds true for a general Hermitian metric $\omega$. This gives a sufficient condition for moderate measures.

Proposition 5.5 If $\hat{\mu}$ is Hölder continuous on $\left\{v \in P S H(\omega): \sup _{X} v=0\right\}$, then it is moderate.

Another sufficient condition for a measure to be moderate, due to Dinh, Nguyen and Sibony [12], is as follows. 
Lemma 5.6 If there exists a Hölder continuous $\omega$-psh function $\varphi$ and a constant $C>0$ such that $\mu \leq C \omega_{\varphi}^{n}$, then $\mu$ is moderate and $\hat{\mu}$ is Hölder continuous on $\mathcal{S}$.

Proof These properties are local by [26, Lemma 1.2] and Lemma 5.3. Therefore, we only prove them in a local coordinate chart. Let $U:=B(x, r) \subset \Omega:=B(x, 2 r)$. Then, we can assume $\mu$ is compactly supported in $U$ and $\mu \leq\left(d d^{c} \varphi\right)^{n}$ for some Hölder continuous plurisubharmonic function $\varphi$ in $\Omega$. By [12, Corollary 1.2] (see also [33, Lemma 2.7, Proposition 2.9]) we get that $\mu$ is moderate and $\hat{\mu}$ is Hölder continuous on $\mathcal{E}_{0}^{\prime}(\Omega)$. Thus, it is also Hölder continuous on $\mathcal{S}$.

Remark 5.7 If $\omega$ is Kähler, then under the assumption of the lemma $\mu$ is indeed Hölder continuous on $\left\{v \in P S H(\omega): \sup _{X} v=0\right\}$. However, due to the torsion terms $d d^{c} \omega$ and $d \omega \wedge d^{c} \omega$ in the general Hermitian case, it seems the Hölder continuity only holds on the smaller set $\mathcal{S}$.

We are ready to prove the Dinh-Nguyen type characterization on Hermitian manifolds.

Theorem 5.8 A positive Radon measure $\mu$ belongs to $\mathcal{H}(\tau)$ and $\hat{\mu}$ is Hölder continuous on $\mathcal{S}$ if and only if there exists a Hölder continuous $\omega$-psh function $u$ and a constant $c>0$ such that

$$
\left(\omega+d d^{c} u\right)^{n}=c \mu .
$$

Proof The second condition implies the first by Lemma 5.6. It remains to show the reverse direction. Theorem 3.1 gives a continuous $\omega$-psh function $u$ and a constant $c>0$ solving the equation. To show that the function $u$ is Hölder continuous we follow the proof of [26, Theorem 1.3]. Note that we used the Hölder continuity of $\hat{\mu}$ on $\mathcal{S}$ and [23, Eq.(1.1)] to get the validity of [26, Lemma 2.8$]$ in the present setting.

The last theorem allows to extend results of Pham [35] and Vu [42] from the Kähler to the Hermitian setting.

Proposition 5.9 Let $\mu$ be a positive Radon measure on X. Assume there exist constants $A, \alpha, t_{0}>0$ such that for every ball $B(x, t) \subset X$,

$$
\mu(B(x, t)) \leq A t^{2 n-2+\alpha} \text { for every } 0<t \leq t_{0} .
$$

Suppose $0 \leq f \in L^{p}(X, d \mu)$ with $p>1$. Assume that $\int_{X} f d \mu>0$. Then, there exist a constant $c>0$ and a Hölder continuous $\omega-$ psh function solving

$$
\left(\omega+d d^{c} u\right)^{n}=c f d \mu .
$$

Proof By Theorem 5.8 it is sufficient to show that $f d \mu$ belongs to $\mathcal{H}(\tau)$ for some $\tau>0$ and that the corresponding functional is Hölder continuous on $\mathcal{S}$. These properties are local. We may assume that supp $\mu \subset U:=B(a, r) \subset \Omega:=B(a, 2 r)$ in $\mathbb{C}^{n}$. By [33, Lemma 2.15, Corollary 2.14] it follows that $\hat{\mu}$ is Hölder continuous on $\mathcal{E}_{0}^{\prime}(\Omega)$, then so is the functional of $f d \mu$. Finally, by [33, Propositon 2.9] we have that $f d \mu$ is moderate.

One example of measures satisfying the assumption of the proposition above is given by the smooth volume form of a smooth hypersurface as in Pham [35].

Corollary 5.10 Let $S$ be a compact smooth real hypersurface in $X$ and $d V_{S}$ is its smooth volume form. Then, for every $0 \leq f \in L^{p}\left(S, d V_{S}\right)$ with $p>1$ and $\int_{S} f d V_{S}>0$, there exist a constant $c>0$ and a Hölder continuous $\omega-$ psh function $u$ solving

$$
\left(\omega+d d^{c} u\right)^{n}=c f d V_{S} .
$$


Later on, $\mathrm{Vu}$ [42] proved the result for a generic CR immersed $C^{3}$-submanifold of $X$. The Kähler assumption in his paper is needed only to use the characterization of [13]. Given our results above we get immediately the statement of his result in the Hermitian setting. Actually, we can also simplify a bit his arguments by using the local Hölder continuity criterion (Lemma 5.3).

Acknowledgements The authors are partially supported by NCN Grant 2017/27/B/ST1/01145. The second author is also partially supported by the start-up Grant G04190056 of KAIST.

Open Access This article is licensed under a Creative Commons Attribution 4.0 International License, which permits use, sharing, adaptation, distribution and reproduction in any medium or format, as long as you give appropriate credit to the original author(s) and the source, provide a link to the Creative Commons licence, and indicate if changes were made. The images or other third party material in this article are included in the article's Creative Commons licence, unless indicated otherwise in a credit line to the material. If material is not included in the article's Creative Commons licence and your intended use is not permitted by statutory regulation or exceeds the permitted use, you will need to obtain permission directly from the copyright holder. To view a copy of this licence, visit http://creativecommons.org/licenses/by/4.0/.

\section{References}

1. Bedford, E., Taylor, B.A.: A new capacity for plurisubharmonic functions. Acta Math. 149, 1-40 (1982)

2. Benelkourchi, S., Jennane, B., Zeriahi, A.: Polya's inequalities, global uniform integrability and the size of plurisubharmonic lemniscates. Ark. Mat. 43, 85-112 (2005)

3. Caffarelli, L., Kohn, J., Nirenberg, L., Spruck, J.: The Dirichlet problem for nonlinear second-order elliptic equations. II. Complex Monge-Ampère, and uniformly elliptic, equations. Commun. Pure Appl. Math. 38(2), 209-252 (1985)

4. Cegrell, U.: Pluricomplex energy. Acta Math. 180(2), 187-217 (1998)

5. Cherrier, P.: Équations de Monge-Ampère sur les variétés Hermitiennes compactes. Bull. Sci. Math. 111(2), 343-385 (1987)

6. Demailly, J.-P.: Measures de Monge-Ampère et caractérisation géométrique des variétés algébraiques affines. Mem. Soc. Math. France (N.S.) 19, 1-124 (1985)

7. Demailly, J.-P., Dinew, S., Guedj, V., Hiep, P., Kołodziej, S., Zeriahi, A.: Hölder continuous solutions to Monge-Ampère equations. JEMS 16(4), 619-647 (2014)

8. Di Nezza, E., Favre, C.: Regularity of push-forward of Monge-Ampère measures. Ann. Inst. Fourier (Grenoble) 68(7), 2965-2979 (2018)

9. Dinew, S.: Pluripotential theory on compact Hermitian manifolds. Ann. Fac. Sci. Toulouse Math. (6) 25(1), 91-139 (2016)

10. Dinew, S.: Lectures on Pluripotential Theory on Compact Hermitian Manifolds. Lecture Notes in Mathematics. Complex Non-Kähler Geometry, vol. 2246. Springer, Berlin (2019)

11. Dinew, S., Kołodziej, S.: Pluripotential Estimates on Compact Hermitian Manifolds. Advanced Lectures in Mathematics, vol. 21. International Press, Boston (2012)

12. Dinh, T.-C., Nguyen, V.-A., Sibony, N.: Exponential estimates for plurisubharmonic functions and stochastic dynamics. J. Differ. Geom. 84(3), 465-488 (2010)

13. Dinh, T.-C., Nguyen, V.-A.: Characterization of Monge-Ampère measures with Hölder continuous potentials. J. Funct. Anal. 266(1), 67-84 (2014)

14. Fang, S., Tosatti, V., Weinkove, B., Zheng, T.: Inoue surfaces and the Chern-Ricci flow. J. Funct. Anal. 271(11), 3162-3185 (2016)

15. Gill, M.: Convergence of the parabolic complex Monge-Ampère equation on compact Hermitian manifolds. Commun. Anal. Geom. 19, 277-303 (2011)

16. Gill, M.: The Chern-Ricci flow on smooth minimal models of general type. Preprint arXiv: 1307.0066v1

17. Guedj, V., Lu, H.-C., Zeriahi, A.: Stability of solutions to complex Monge-Ampère flows. Annales de l'Institut Fourier 68(7), 2819-2836 (2018)

18. Guedj, V., Zeriahi, A.: The weighted Monge-Ampère energy of quasiplurisubharmonic functions. J. Funct. Anal. 250(2), 442-482 (2007)

19. Kołodziej, S.: Some sufficient conditions for solvability of the Dirichlet problem for the complex MongeAmpère operator. Ann. Polon. Math. 65, 11-21 (1996)

20. Kołodziej, S.: The complex Monge-Ampère equation. Acta Math. 180, 69-117 (1998) 
21. Kołodziej, S.: The Monge-Ampère equation on compact Kähler manifolds. Indiana Univ. Math. J. 52, 667-686 (2003)

22. Kołodziej, S.: The complex Monge-Ampère equation and pluripotential theory. Mem. Am. Math. Soc. 178, 64 (2005)

23. Kołodziej, S.: Hölder continuity of solutions to the complex Monge-Ampère equation with the right hand side in $L^{p}$. The case of compact Kähler manifolds. Math. Ann. 342, 379-386 (2008)

24. Kołodziej,S., Nguyen, N.-C.: Weak Solutions to the Complex Monge-Ampère Equation on Hermitian Manifolds. Analysis, Complex Geometry, and Mathematical Physics: In Honor of Duong H. Phong, Contemporary Mathematics, vol. 644. American Mathematical Society, Providence, pp 141-158 (2015)

25. Kołodziej, S., Nguyen, N.C.: Weak solutions of complex Hessian equations on compact Hermitian manifolds. Compos. Math. 152(11), 2221-2248 (2016)

26. Kołodziej, S., Nguyen, N.C.: Hölder continuous solutions of the Monge-Ampère equation on compact Hermitian manifolds. Ann. Inst. Fourier (Grenoble) 68(7), 2951-2964 (2018)

27. Kołodziej, S., Nguyen, N.C.: Stability and regularity of solutions of the Monge-Ampère equation on Hermitian manifolds. Adv. Math. 346, 264-304 (2019)

28. Kołodziej, S., Nguyen, N.C.: A remark on the continuous subsolution problem for the complex MongeAmpère equation. Acta Math. Vietnam 45, 83-91 (2020)

29. Kołodziej, S., Tosatti, V.: Morse-type integrals on non-Kähler manifolds. arXiv:1906.09614. To appear in Pure Appl. Math. Q (2021)

30. Lu, C.-H., Phung, T.-T., To, T.-D.: Stability and Hölder regularity of solutions to complex Monge-Ampère equations on compact Hermitian manifolds. arXiv: 2003.08417. to appear in Annales de l'Institut Fourier

31. Nie, X.: Weak solution of the Chern-Ricci flow on compact complex surfaces. Math. Res. Lett. 24(6), 1819-1844 (2017)

32. Nguyen, N.-C.: The complex Monge-Ampère type equation on compact Hermitian manifolds and applications. Adv. Math. 286, 240-285 (2016)

33. Nguyen, N.-C.: On the Hölder continuous subsolution problem for the complex Monge-Ampère equation. Calc. Var. Partial Differ. Equ. 57(1), Art. 8, 15 pp (2018)

34. Nguyen, N.-C.: On the Hölder continuous subsolution problem for the complex Monge-Ampère equation. II. Anal. PDE 13(2), 435-453 (2020)

35. Pham, H.-H.: Hölder continuity of solutions to the Monge-Ampère equations on compact Kähler manifolds. Ann. Inst. Fourier (Grenoble) 60(5), 1857-1869 (2010)

36. Tosatti, V., Weinkove, B.: The complex Monge-Ampère equation on compact Hermitian manifolds. J. Am. Math. Soc. 23, 1187-1195 (2010)

37. Tosatti, V., Weinkove, B.: Plurisubharmonic functions and nef classes on complex manifolds. Proc. Am. Math. Soc. 140(11), 4003-4010 (2012)

38. Tosatti, V., Weinkove, B.: The Chern-Ricci flow on complex surfaces. Compos. Math. 149(12), 21012138 (2013)

39. Tosatti, V., Weinkove, B.: On the evolution of a Hermitian metric by its Chern-Ricci form. J. Differ. Geom. 99, 125-163 (2015)

40. Tosatti, V., Weinkove, B., Yang, W.: Collapsing of the Chern-Ricci flow on elliptic surfaces. Math. Ann. 362, 1223-1271 (2015)

41. Tô, D.: Regularizing properties of complex Monge-Ampère flows II: Hermitian manifolds. Math. Ann. 372(1-2), 699-741 (2018)

42. Vu, D.-V.: Complex Monge-Ampère equation for measures supported on real submanifolds. Math. Ann. 372(1-2), 321-367 (2018)

43. Vu, D.-V.: Equilibrium measures of meromorphic self-maps on non-Kähler manifolds. Trans. Am. Math. Soc. 373(3), 2229-2250 (2020)

44. Yau, S.-T.: On the Ricci curvature of a compact Kähler manifold and the complex Monge-Ampère equation. Commun. Pure Appl. Math. 31, 339-411 (1978)

45. Zheng, T.: The Chern-Ricci flow on Oeljeklaus-Toma manifolds. Can. J. Math. 69(1), 220-240 (2017)

Publisher's Note Springer Nature remains neutral with regard to jurisdictional claims in published maps and institutional affiliations. 